\title{
Interesting developments at the nanoparticle-protein interface: implications for next generation drug delivery
}

\author{
"If these materials [nanoparticles] represent an important new \\ avenue of therapeutic delivery that is under concerted development, \\ then we must understand how they work! Clearly, this is an area \\ which is ripe for research with a strong potential payoff."
}

First draft submitted: 20 May 2016; Accepted for publication: 26 May 2016; Published online: 22 July 2016

Keywords: bioconjugate $\bullet$ delivery $\bullet$ drug $\bullet$ enzyme $\bullet$ nanoparticle $\bullet$ nanotechnology - quantum dot • theranostics

All of us are familiar with the growing chorus lamenting how hard it is to develop the next generation of therapeutics. Cost is invariably at the top of the list of complaints, but this is soon followed by the technical challenges which, in reality, may be harder to overcome. Here the issues encompass toxicity profiles, solubility, targeting, tolerance, cross-reactivity, clearance and so on [1]. One fascinating option put forth to address this significant problem seems almost the stuff of science fiction - that is switching away from the classical paradigm of systemic administration to one based on theranostics - and especially in the form of nanoparticle mediated drug delivery $[2,3]$. Several high-profile research foci currently coalesce around this theme. Conceptually, the idea is that a nanoparticle (NP) will be utilized as a central scaffold and targeted delivery vehicle. Antibodies, aptamers or similarly functional biologicals are decorated on the NP surface to provide in vivo targeting to a specific wound, infection site or cancer type, for example. Cell penetrating peptides may also be added to help with cellular uptake [4]. The NP will then carry a primary therapeutic cargo either internally or on its surface. Critically, this therapeutic may be extremely toxic, which would preclude its systemic administration or it may lack solubility and be shuttled by the NP. The NP may further act as a contrast agent based on its own intrinsic properties (e.g., magnetic or radio-labeled) to allow for imaging especially when it is concentrated into a tumor mass [5]. Cumulatively, this sounds like a complex nanoscale machine more reminiscent of a futuristic science fiction film such as The Fantastic Voyage than the generalized design for a new class of therapeutics. There are many more detailed discussions of this therapeutic strategy available $[2,3,6]$.

A majority of the currently available drugs on the market target enzymes along with a plethora of other active cellular proteins include channels, transporters and the like who can also be inhibited or modulated in a therapeutic manner akin to enzymes. Furthermore, enzymes themselves constitute a class of therapeutics for replacement type therapies [7]. Considering an NP displaying an enzyme or a drug in the context of nanoparticle mediated drug delivery brings us to a critical issue that is only now starting to be realized. That is, how do enzymes and other proteins function at an NP interface? This has been the subject of much of our research over the last approximately

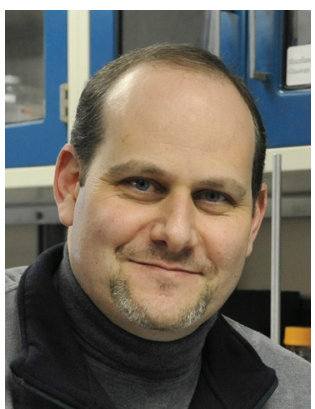

Igor L Medintz

'Senior Scientist for Biosensors \& Biomaterials, Center for Bio/Molecular Science \& Engineering, Code 6907, US Naval Research Laboratory, 4555 Overlook Ave, SW, Washington, DC 20375, USA

Igor.medintz@nrl.navy.mil 
5 years and here we discuss some aspects of it from a conceptual view. The activity of enzymes on bulk planar surfaces such as a slide is generally well understood and encompasses aspects of a laminar environment [8]. In contrast, when attaching an enzyme to an NP, the interface is now reduced to the nanoscale while the entire assembly still maintains the ability to diffuse in solution albeit at a much slower rate than that of a free enzyme. For these purposes, we utilize a loose definition of an NP as being a solid (e.g., gold) or softer material (e.g., polymersome) and $\leq 100 \mathrm{~nm}$ in diameter. Since attaching enzymes to planar surfaces usually results in loss of activity, and putting aside issues arising from improper attachment of the enzyme to the NP, at a first pass one would expect that enzyme attachment to NPs would also lead to a loss of activity [8]. Interestingly, the results are quite the opposite and somewhat counterintuitive. Attaching enzymes, or substrates, to NP surfaces in many cases results in what appears to be augmented or enhanced activity. This is not just an anecdotal observation, it is estimated that there are now more than 200 accumulated reports of such enhancement in the literature [8-10]. Initial research into this materials configuration was driven by a desire to create more robust biosensors, to provide reagents for commercial biocatalytic applications and interest in creating cell-free synthetic biology tools. It now appears that such NP attachment may also improve long-term enzyme stability similar to the effects often seen with planar surfaces.

So what exactly is going on? Many different phenomena were initially suggested as being causative, either individually or collectively, including higher avidity, confinement effects, substrate trapping, optimal enzyme orientation, shielding, colocalization, optimized substrate trajectories and so on [8]. The diversity in this list is not directly reflective of the underlying physical processes but rather serves to indicate just how little is known about this phenomena. Given the complexity inherent to these multicomponent hybrid systems, our focus has been on trying to simplify the systems down to one variable to make them more tractable to experimental elucidation.

Analyzing substrate decorated around an NP was easier to investigate and so this was pursued first. For this, dye-labeled peptidyl substrates were ratiometrically attached to luminescent semiconductor quantum dots (QDs) to monitor trypsin proteolytic activity by FRET as a model system [11]. The only variable modified here was the ratio of peptide-substrate attached to the QDs. Three- to fivefold enhancements in enzymatic activity $\left(\mathrm{k}_{\text {cat }}\right)$ and efficiency $\left(\mathrm{k}_{\mathrm{cat}} / \mathrm{K}_{\mathrm{m}}\right)$ were observed for on-QD substrate as compared directly with equivalent no-QD control samples. Modeling suggested a 'hopping' mode of substrate consumption where the protease interacted with an initial peptide on a QD, then consumed all the remaining peptide on that QD before diffusing off to the next encounter. Most simplistically, the localized high-avidity display of substrate in conjunction with long enzyme dwell times around the QD contributed to enhanced activity. As expected, the enzymatic activity did not match to any classical Michaelis-Menten (MM) models. Clearly, how the enzyme interacts with the combined peptide-NP surface environment that it encounters will strongly influence how this type of activity proceeds. Examples where the protease is repulsed by like charges on the NP have indeed confirmed this [12].

Analyzing the activity of NPs displaying enzymes also provided evidence for enhanced activity [13-15]. Here a system consisting of phosphotriesterase (PTE, $37 \mathrm{kD}$ ) displayed around QDs proved extremely insightful [15]. In this case, a fourfold increases in initial rate and twofold increases in enzymatic activity were observed over a range of QD-PTE ratios. A series of in-depth diagnostic assays revealed that the higher activity in this case was due to an acceleration in the enzyme-product dissociation rate which is the enzyme's postulated rate limiting step. This presumably arose from the markedly different microenvironment found in the localized QD-PTE hydration layer. Supporting this notion, a recent report confirms that structural rearrangement of the localized environment around colloidal NPs is a universal phenomenon regardless of what the actual solvent is [16]. We have even found a significant enhancement when working with the large multimeric $\beta$-galactosidase enzyme and QDs [17]. Given the large size of this tetrameric enzyme (465 $\mathrm{kD})$, the QDs were functionally decorated around the enzyme instead of the converse configuration. Interestingly, as $\beta$-gal is essentially a diffusion-limited enzyme, the interpretation from these results suggests substrate sequestration around the assembly as the being the cause of enhancement. Although mechanistically different from the above PTE example, the localized substrate accumulation is still postulated to arise from the same unique structured environment that surrounds the NP-enzyme construct. It is important to point out at this point that there are currently almost no metrologies or analytical techniques available that can provide any type of direct data on the physical properties and characteristics of what is essentially a very complex nanoscale interface between two material types [18]. Physical properties that are expected to vary radically in this environment include ionic concentrations, viscosity, polarity, solvation, localized density and molecular $\mathrm{pK}_{\mathrm{a}} \mathrm{s}$ [19]. Other phenomena that may also contribute here include phase separation and boundary 
layers. This suggests that this localized environment may vary quite significantly based on the type of NP used, its surface chemistry and enzyme size along with other undetermined factors. Nevertheless, the common recurrent theme is that the net result in most cases is some type of enhanced enzymatic activity.

So coming back full circle, what does this all mean for therapeutic delivery based on using NPs even as just a targeted carrier? It is far too early to make definitive statements at this stage, but some generalities will probably hold true. Reminiscent of many other things we encounter in life, developing this type of therapeutic material will be far more complicated than initially anticipated but for reasons that are not so obvious and perhaps even beneficial. For example, based on the above early forays into this field, it is not out of the realm of possibility to suggest that some of the NP-based therapeutic materials may be far more potent than expected or function in other unanticipated but still useful ways. This would argue, in turn, that less material may be needed for an effective therapeutic dosage. What is currently required is not only a characterization of how these materials function, but more importantly, a fundamental understanding or model of how they work. This will be extremely challenging beyond just developing new analytical techniques as there are far more variables involved in assembling these types of materials and introducing them into a biological environment. For optimal function, the biologicals - be they drug or enzyme - must be attached to the NP in a completely controlled, reproducible manner which is exceedingly hard to consistently achieve [20,21]. There is also the unresolved issue of corona formation where NP-based

\section{References}

1 Web Collection Ten years in drug discovery. www.nature. com/nrd/collections/10/index.html

2 Petros RA, DeSimone JM. Strategies in the design of nanoparticles for therapeutic applications. Nat. Rev. Drug Discov. 9, 615-627 (2010).

3 Delehanty JB, Boeneman K, Bradburne CE et al. Quantum dots: a powerful tool for understanding the intricacies of nanoparticle-mediated drug delivery. Expert Opin. Drug Deliv. 6, 1091-1112 (2009).

4 Field LD, Delehanty JB, Chen YC et al. Peptides for specifically targeting nanoparticles to cellular organelles: Quo vadis? Acc. Chem. Res. 48, 1380-1390 (2015).

5 Sapsford KE, Algar WR, Berti L et al. Functionalizing nanoparticles with biological molecules: developing chemistries that facilitate nanotechnology. Chem. Rev. 113, 1904-2074 (2013).

6 Min YZ, Caster JM, Eblan MJ et al. Clinical translation of materials are fouled by a myriad of molecules found in vivo which mask much of the surface [22]. Lastly, there is the issue of targeted drug release from the NP only when and where it is required in vivo [23]. There is a growing list of US FDA-approved 'nanoparticle' therapeutics, but they pale in comparison to the sophistication being described here [24]. If these materials represent an important new avenue of therapeutic delivery that is under concerted development, then we must understand how they work! Clearly, this is an area which is ripe for research with a strong potential payoff. It certainly represents a prime example to be tackled by an amalgam of interdisciplinary researchers as it requires a broad swath of expertise ranging from biochemistry to materials science and pharmacology. Beyond just challenges, we can also expect that far more opportunities will be unveiled as we delve into this exciting interface to try and unravel these complex phenomena [25].

\section{Acknowledgement}

The author acknowledges the Joint Science and Technology Office of the Defense Threat Reduction Agency and US Naval Research Laboratory Nanosciences Institute.

\section{Financial \& competing interests disclosure}

The author has no relevant affiliations or financial involvement with any organization or entity with a financial interest in or financial conflict withthe subject matter or materials discussed in the manuscript. This includes employment, consultancies, honoraria, stock ownership or options, expert testimony, grants or patents received or pending, or royalties.

No writing assistance was utilized in the production of this manuscript.

nanomedicine. Chem. Rev. 115, 11147-11190 (2015)

7 Law V, Knox C, Djoumbou Y et al. DrugBank 4.0: shedding new light on drug metabolism. Nucleic Acids Res. 42, D1091-1097 (2014).

8 Johnson BJ, Algar WR, Malanoski AP et al. Understanding enzymatic acceleration at nanoparticle interfaces: approaches and challenges. Nano Today 9, 102-131 (2014)

9 Ansari SA, Usain Q. Potential applications of enzymes immobilized on/in nano materials: a review. Biotechnol. $A d v$. 30, 512-523 (2012)

10 Ding S, Cargill AA, Medintz IL et al. Increasing the activity of immobilized enzymes with nanoparticle conjugation. Curr. Opin. Biotechnol. 34, 242-250 (2015).

11 Algar WR, Malonski AP, Deschamps JR et al. Proteolytic activity at quantum dot-conjugates: kinetic analysis reveals enhanced enzyme activity and localized interfacial 'hopping'. Nano Lett. 12, 3793-3802 (2012).

12 Diaz SA, Malanoski A, Susumu K et al. Probing the kinetics of quantum dot-based proteolytic sensors. Anal. Bioanal. 
Chem. 407, 7307-7318 (2015).

13 Claussen JC, Malanoski A, Breger JC et al. Probing the enzymatic activity of alkaline phosphatase within quantum dot bioconjugates. J. Phys. Chem C 119, 2208-2221 (2015).

14 Breger JC, Walper SA, Oh E et al. Quantum dot display enhances activity of a phosphotriesterase trimer. Chem. Commun. 51, 6403-6406 (2015).

15 Breger JC, Ancona MG, Walper S et al. Understanding how nanoparticle attachment enhances phosphotriesterase kinetic efficiency. ACS Nano 8, 8491-8503 (2015).

16 Zobel M, Neder RB, Kimber SAJ. Universal solvent restructuring induced by colloidal nanoparticles. Science 347, 292-294 (2015).

17 Brown CW, Oh E, Hastman DA et al. Kinetic enhancement of the diffusion-limited enzyme beta-galactosidase when displayed with quantum dots. RSC Adv. 5, 93089-93094 (2015).

18 Sapsford KE, Tyner K, Dair B et al. Analyzing nanomaterial bioconjugates: a review of current and emerging techniques for purification and characterization. Anal. Chem. 83, 4453-4488 (2011).

19 Pfeiffer C, Rehbock C, Huhn D et al. Interaction of colloidal nanoparticles with their local environment: the (ionic) nanoenvironment around nanoparticles is different from bulk and determines the physico-chemical properties of the nanoparticles. J. R. Soc. Interface 11, 20130931 (2014)

20 Medintz IL. Universal tools for biomolecular attachment to surfaces. Nat. Mater. 5, 842 (2006).

21 Walper SA, Turner KB, Medintz IL. Enzymatic bioconjugation of nanoparticles: developing specificity and control. Curr. Opin. Biotechnol. 34, 232-241 (2015).

22 Corbo C, Molinaro R, Parodi A et al. The impact of nanoparticle protein corona on cytotoxicity, immunotoxicity and target drug delivery. Nanomedicine 11, 81-100 (2016)

23 Delehanty JB, Breger JC, Boeneman Gemmill K et al. Controlling the actuation of therapeutic nanomaterials: enabling nanoparticle-mediated drug delivery. Ther. Deliv. 4, 1411-1429 (2013).

24 Eifler AC, Thaxton CS. Nanoparticle therapeutics: FDA approval, clinical trials, regulatory pathways, and case study. Methods Mol. Biol. 726, 325-338 (2011)

25 Dennis AM, Delehanty JB, Medintz IL. Emerging physicochemical phenomena along with new opportunities at the biomolecular nanoparticle interface. J. Phys. Chem. Lett. 7, 2139-2150 (2016). 